\title{
Teaching Embedded System Design
}

\author{
K. Ferens \\ Department of Electrical and Computer Engineering \\ University of Manitoba \\ Winnipeg, Manitoba, Canada R3T5V6 \\ ferens@ee.umanitoba.ca
}

\begin{abstract}
For the first time in the Department of Electrical and Computer Engineering at the University of Manitoba, a $100 \%$ hands-on course was implemented in the winter term of the 2011/2012 year. This course ECE 3730 Embedded System Design was introduced into the curriculum and designed specifically to correct an imbalance between computer and electrical engineering student pre-requisites; to address students studying only for the exam problem; and to directly assess student performance particularly in the CEAB attributes of Design, Investigation, Problem Analysis, and Tools.
\end{abstract}

Keywords-Embedded system, engineering learning outcomes, CEAB attributes.

\section{Introduction}

Today, the scope of applications of embedded systems is continuing to increase with applications that range from simple toys to complex electronic devices such as smart phone, signal analyzers, parallel implementations, and real time systems. In the very near future, everything of material significance will be outfitted with an embed microcontroller. Given the demand and future certainties, computer engineering academics around the world have increased their focus on teaching embedded systems to improve the methods of teaching embedded systems and learning and discovering new, novel, and innovative methods of teaching embedded systems [1] [2] [3].

The Department of Electrical and Computer Engineering (ECE) at the University of Manitoba has an embedded focus area, which, aims to guide students along a path that imparts the analysis, design, and implementation of algorithms, hardware, and software that makeup embedded systems. The focus area specifies a stream of required courses:

1. ECE 2220 Digital Logic

2. ECE 3610 Microprocessing Systems

3. ECE 3740 Systems Engineering Principles 1

4. ECE 3760 Digital System Design 1

5. ECE 4240 Microprocessor Interfacing

6. ECE 4740 Digital System Implementation

While the courses in this stream complemented the natural progression of computer engineering students in the
Department, the electrical engineering students found difficulty with "ECE 3740 Systems Engineering Principles 1," because the computer engineering students had a broader background in computer related course. To correct this imbalance, a new course was introduced ECE 3730 Embedded System Design, which catered to the prerequisite deficit of electrical students. In addition to satisfying the prerequisite requirement, the Embedded System Design course also had to satisfy new outcomes based assessment requirements.

The Canadian Engineering Accreditation Board (CEAB) [1] has established new and additional accreditation criteria for undergraduate programs in Canadian Engineering educational institutions. These new accreditation criteria are focused on outcomes based assessment, and they state:

"The institution must demonstrate that the graduates of a program possess the attributes under the following headings. The attributes will be interpreted in the context of candidates at the time of graduation."

\section{A knowledge base for engineering \\ 2. Problem analysis \\ 3. Investigation (Validation) \\ 4. Design \\ 5. Use of engineering tools \\ 6. Individual and teamwork \\ 7. Communication skills \\ 8. Professionalism \\ 9. Impact of engineering on society and the environment \\ 10. Ethics and equity \\ 11. Economics and project management \\ 12. Life-long learning}

In the past, curricula designers focused on teaching content and designing labs to complement the concepts taught in class. To align with the Washington Accord, the CEAB requires the engineering institutions show that their graduates possess the 12 attributes.

Taking advantage of the opportunity to design a new course and leveraging the new $\mathrm{CEAB}$ attribute requirements, the design of the ECE 3730 Embedded System Design course was specifically targeted towards directly training and assessing student performance particularly in the CEAB attributes of Design, Investigation, Problem Analysis, and Tools. 
In addition to satisfying the CEAB attribute requirement, the Embedded System Design course also took the opportunity to align itself with student behavior. As the work done by [5] suggests, students tend to focus on trying to learn the content of past tests and exams. The design of the ECE Embedded System Design course leveraged that knowledge by giving students projects throughout the semester and informing the students that the exam will be similar to the projects. The projects were designed to cover the major concepts and areas of embedded systems, so that if a student knows how to do a project, the student will do well on the exam; will learn the course; and will automatically exhibit the targeted $\mathrm{CEAB}$ attributes.

\section{Course Design}

The ECE 3730 Embedded Systems Design course was designed as $100 \%$ hands-on. At the beginning of the course, each student was given a set of microcontroller based parts, which were to be used to implement a stream of projects in the course. The parts included a Microchip PIC 16F877A microcontroller, rotary switch, seven segment display unit, LED display unit, Digital to Analog Converter (ADC8562), First-In-First-Out (FIFO) memory (IDT7200), and GPIO Expander with serial interface (MCP23017). Students were also instructed to download and install Microchip's development software called MPLAB, integrated development environment (IDE). In addition students were instructed to use Microchip's debugger, called ICD3, to allow them to upload their programs onto the microcontroller and to allow debugging, such as placing breakpoints and stepping through their programs.

The course was structured around 5 projects. Each project had a pre-assignment and a post-assignment. The pre- and postassignments were performed outside of the laboratory (at home or wherever), and the main project was performed in the lab. The projects were design to be implemented in an incremental fashion, to teach the students about incremental design and the divide-and-conquer design principle. Students created separate MPLAB projects for each of the assignments and main projects. As well, students suffixed their projects with versions numbers, and thus students gained experience in the motivation and importance of version control.

The lectures provided theory and examples of applications of the theory. The main topics were

1. C language Basics

2. microcontroller architecture, programming model, memory map, pipelining, and peripherals (ADC, GPIO, Timers, Comparators)

3. Analog to Digital Conversion (ADC) and Digital to Analog Conversion (DAC)

4. Buffering (single, double, circular, and FIFO)

5. Switches (debouncing)
6. Displays (multiplexed seven segment, liquid crystal display (LCD))

7. Modeling (flowcharts, Unified modeling Language (UML), state diagrams)

8. C Language Data Structures and Pointers (Arrays, pointers, structures, structures for embedded systems)

9. General Purpose Input Ouput (GPIO) Expander

10. Serial Communications (Basic Serial, RS232 (ASCI and UART), Serial Peripheral Interface (SPI), and Inter-Integrated Circuit Communications $\left(\mathrm{I}^{2} \mathrm{C}\right)$

11. Counter and Timers (Input and Output Capture)

12. Motors for Embedded Systems (Stepper Motors)

13. Logic Devices (Open Drain and open Collector)

The level of difficulty of the topics presented in the course ranged from relatively simple (Application of Rotary Switch with 7-segment Display); to average difficulty (Waveform Generation); to relatively difficult (Interfacing an LCD with a serial interface GPIO Expander).

For example, Fig. 1 shows an oscilloscope capture of the output of a switch when it was thrown to the "on" position. As can been seen, the voltage bounces through several on/off states. The lecture notes discussed switch bouncing as well gave the circuit analysis and implementation using the PIC 16F877A microcontroller.

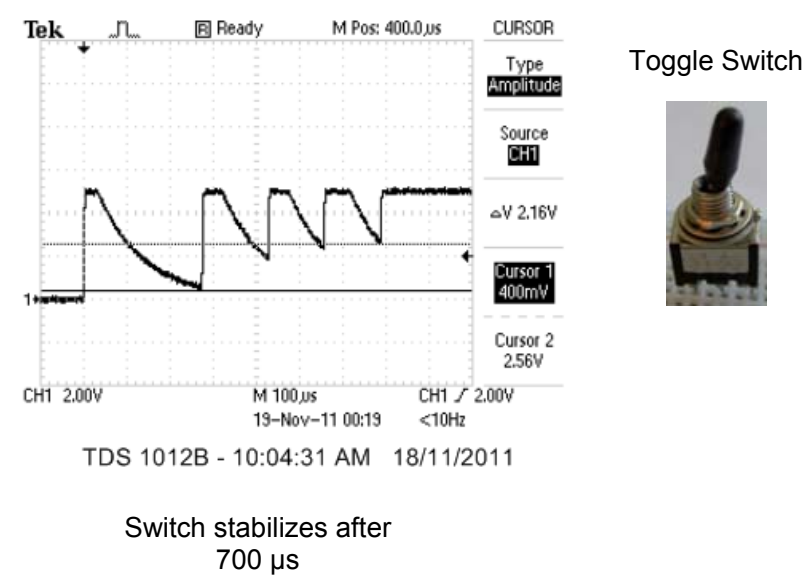

Fig. 1. Oscilloscope capture of a bouncing switch.

The five projects of the course (with pre- and postassignments for each) were as follows:

1. Rotary Encoder with Multiplexed 7-Segment Display

2. Arbitrary Waveform Generator

3. LCD Interface with GPIO Expander and SPI

4. Multiprocessor Parallel Data Communication Using a Hardware FIFO

5. Stepper Motor 
Fig. 2 shows another example: a waveform generator.

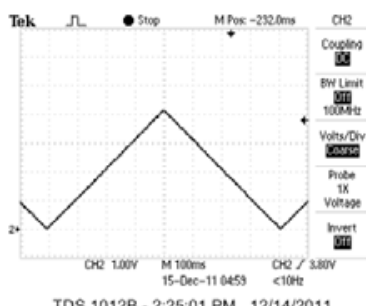

TOS 1012B - 2:25:01 PM 12/14/2011

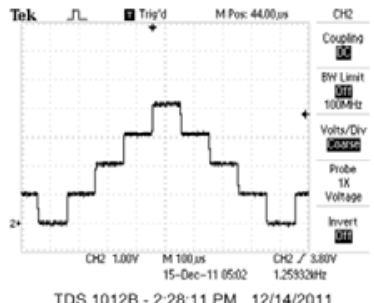

TOS 1012B - 2:28:11 PM 12/14/2011

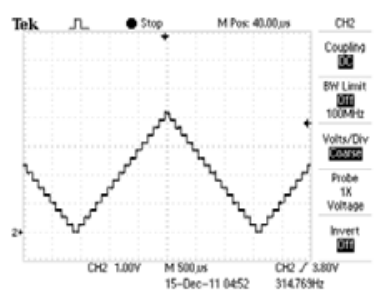

TOS 1012B - 2:17:57 PM 12/14/2011

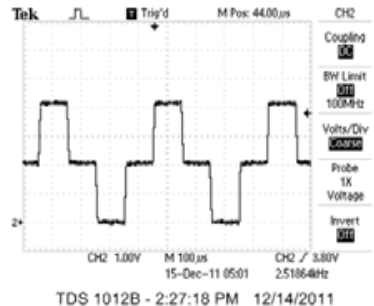

Fig. 2. Oscilloscope captures of a triangular waveform generation with different sample output times.

\section{CEAB Attribute Targets}

The design of the ECE 3730 Embedded System Design course was specifically targeted towards directly training and assessing student performance particularly in the CEAB attributes of Design, Investigation, Problem Analysis, and Tools.

\section{Design}

The design component of this course was spread through five embedded systems projects (ESP1 to ESP5), one midterm, and the final exam. Students designed microcontroller based solutions for various simple embedded problems throughout the entire course. The course was a hands-on design of embedded systems using microcontrollers. Each project consisted of two assignments and one laboratory. The projects were structured to enable students to practice incremental development and design in each of the projects. In each of the projects, students were given the necessary components (take home) to implement the design (MPLAB IDE, ICD3 debugger, PIC16F877A microcontroller, rotary encoder (switch), dual seven segment display unit, LCD, FIFO, GPIO Port expander, SPI, and project board). They wired together the components on a project board, and developed, debugged, and tested their code on the prototype project-boards using the MPLAB IDE. The final exam and midterm were completely hands-on and were taken and performed in the laboratory. Students were given a short description the problem and the necessary components. In each of their designs, students discovered and evaluated different design alternatives, and chose the option that most closely satisfied the requirements of the problem. For example, the interface of the rotary encoder and dual segment display may be implemented using two threads, one interrupt routine for the rotary switch and the foreground routine for display on the segment display; other designs are possible, each of which had advantages and disadvantages. Furthermore, students practiced design using several different C-language programming constructs, such as while and for loops, if-thenelse, switch, structures, pointers, function pointers, and linked lists. Students evaluated the advantages and disadvantages of using such constructs, and chose the construct based on efficiency and optimization of code size, complexity or speed, depending on the problem requirements.

\section{Investigation}

Students practiced investigation through the five embedded systems projects (ESP1 to ESP5), one midterm, and the final exam. Students used and developed their debugging skills to get their code working properly. In particular, students learned how to investigate why their programs were not working as intended. Breakpoints in the software were placed at strategic locations. Students carefully analyzed the symptoms of the problem, determined what the program was supposed to be doing at each point in their programs, and then determined a strategic point to place a breakpoint. Once the program had stopped at the breakpoint, students then analyzed the contents of the registers and memory locations to determine if they contained the expected values. Furthermore, they stepped through each instruction following the breakpoint to determine at which instruction the problem began to manifest itself.

In addition, they practiced interacting with the hardware while stepping through their code. For example, to determine why they were unable to write to the FIFO correctly, they placed a breakpoint in the interrupt service routine (ISR). At this point they analyzed the output control signals on an oscilloscope, and found that the Read signal was active while the ISR was trying to write data to the FIFO with the Write signal active as well. Since the FIFO read and write functions shared the same bus, the data the ISR attempted to write to the FIFO contended with the Data the FIFO was simultaneously writing to the bus. Consulting the FIFO data sheet, students learned that the main thread was being interrupted before it had the chance to de-assert the Read signal. Through this complex investigation and analysis, students learned how to solve the problem.

In addition, students learned how to insert visualization code in their programs to visualize on the oscilloscope the states of their running programs.

For each one of the projects, students developed unit tests as incremental development. The unit tests investigated the functionality of the device to determine proper operation. For example, student performed unit test on the Digital to Analog converter to determine the proper amount of settling time. Students captured oscilloscope screen shots of their investigations, analyzed them, and came to logical conclusions. 


\section{Problem Analysis}

Throughout the course, students were trained on an iterative process of software engineering, which consisted of domain analysis; requirements gathering, review and analysis; modeling, translation and coding; and testing. Students were given short, high-level descriptions of a problem to be solved. The actual requirements were buried in the description, and the students used a software engineering process to uncover them. Students first acquired an understanding of the problem domain. Once a moderate understanding of the domain was achieved, students were taught to analyze the problem statement, and then this is followed by gathering the requirements from the stakeholders. Once the statements from the stakeholders were obtained, then students reviewed and analyzed the statements to properly form them into statements of requirements, which were unambiguous, verifiable, identifiable, safe, consistent, and realistic. Through the domain analysis and requirements phases of the iterative procedure, students used appropriate knowledge and skills to identify, formulate, analyze, and solve complex engineering problems.

Students practiced this software engineering process in the labs, assignments, tests, and final exam. For example, in the assignments and labs, students determined the requirements for "determine the throughput when noise is injected into the FIFO communications circuit." Students uncovered several hidden requirements, which were not mentioned in the problem statement, such as point of throughput measurement and point of noise injection.

\section{Final Exam and Midterm Test}

The final exam and midterm test were 100\% "hands-on," and were performed entirely in the laboratory. In the exam, each student was assigned a development workstation, which had the MPLAB integrated development environment (IDE) pre-installed and ready for use. In addition, the Tektronix oscilloscopes with USB connection to the workstation were provided. Additional software on the workstation allowed students to capture oscilloscope screens for presentation of their results. Students brought a pre-wired microcontroller circuit project board into the exam (laboratory). While the circuit diagram was given one week prior to the exam, students were not informed of the actual exam question until the time of the exam (9:00 AM, 12 April 2012). Fig. 3 shows a student's hardware setup for the exam.

\section{Conclusions}

This paper presented a preliminary study of the impact of $100 \%$ hands-on computer engineering course on embedded system design. This course was structured around 5 projects which had pre- and post-assignments as well the main project, which was performed in the lab. The term test and final exam were implemented $100 \%$ hands-on and students performed the exams in the lab. The students in this course were assessed directly in terms of the CEAB attributes of Design, Investigation, Problem Analysis, and Tools. It was found that marking the tests and exams were very telling of the students' performance. It was very clear if the student really understood the material and knew how to do the question. No question about it.

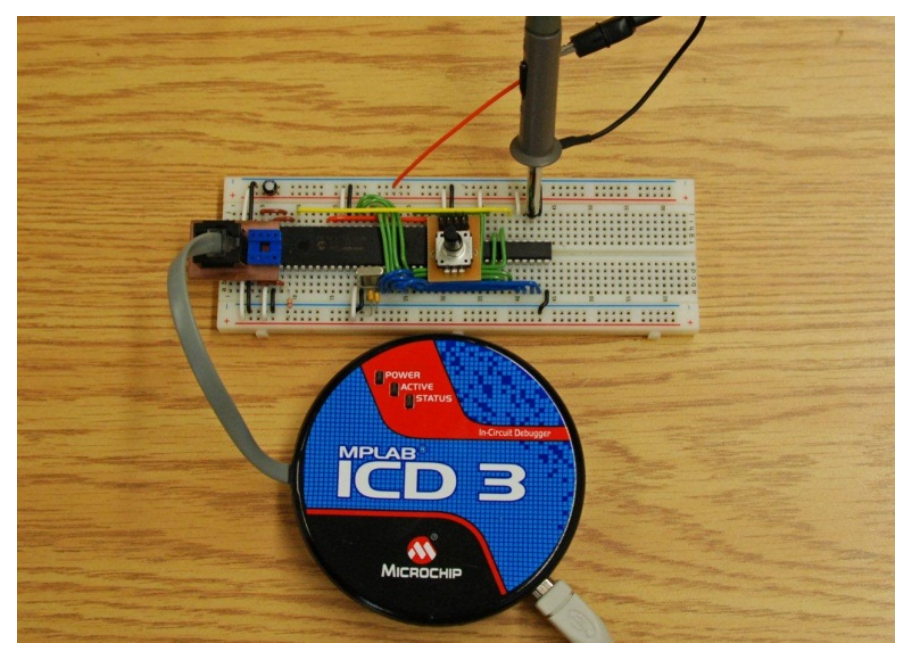

Fig. 3. Hardware setup for the waveform generation examination question (with permission from M. McQuaker).

\section{References}

[1] D. T. Rover, R. A. Mercado, Z. Zhang, M. C. Shelley and D. S. Helvick, "Reflections on Teaching and Learning in an Advanced Undergraduate Course in Embedded Systems," IEEE Trans. Educ, vol. 51, no. 3, pp. 400-412, 2008.

[2] R. S. Selvakuma, K. H. Ghazali, N. M. Nik Yusoff and H. AbdulAziz, "Learning Embedded Systems with MultiMicrocontroller Evaluation Tool," in International Conference on Electrical, Control and Computer Engineering, Pahang, Malaysia, 2011.

[3] K. Ferens, M. R. Friesen and S. Ingram, "Impact Assessment of a Microprocessor Animation on Student Learning and Motivation in Computer Engineering," IEEE Trans. on Education, vol. 50, no. 2, pp. 118-128, 2007.

[4] E. Canada, "Canadian Engineering Accreditation Board Accreditation Criteria and Procedures report 2011," [Online]. Available: http://www.engineerscanada.ca/files/w_Accreditation_Crit eria_Procedures_2011.pdf. [Accessed 8 April 2012].

[5] G. Scott and K. W. Yates, "using successful graduates to improve the quality of undergraduate engineering programmes," European Journal of Engineering Education, vol. 27, no. 4, pp. 363-378, 2002. 